\title{
ACOUSTICS2008/1026 Nanoacoustic waves in nanomaterials
}

\author{
J. I Dijkhuis and P. J S Van Capel \\ Utrecht University, Ornsteinlaboratorium, Princetonplein 1, 3508TA Utrecht, Netherlands
}

We discuss the formation and detection of acoustic soliton trains in crystal slabs. High-amplitude amplitude strain pulses are generated by impact of intense femtosecond optical pulses on a metallic film and injected into the crystal slab. Nonlinear acoustic propagation leads to the formation of shock fronts ( $\mathrm{N}$-waves), that, in absence of viscous damping and by virtue of dispersion, may develop into soliton trains at the leading edge and high frequency tails at the trailing edge. Interferometric pump-probe optical experiments are discussed to directly detect the ultrafast surface displacements when a soliton is reflected at the surface of the crystal slab. Finally, we experimentally prove that these acoustic solitons are capable of impulsively exciting $\mathrm{THz}$ transitions in electronic centers in solids. The coherent terahertz acoustic pulse trains are applied to manipulate the optical response of two-dimensional excitons in a III-V quantum well on the ultrafast timescale. By virtue of the deformation potential, the coherent exciton emission becomes strongly "chirped" when the acoustic pulse train passes the quantum well. This yields the prospect to perform pump-probe terahertz acoustic experiments with nanoacoustic waves in semiconductor nanomaterials.

Keywords: acoustic solitons, terahertz acoustics, semiconductors, quantum wells Technical area: Physical Acoustics (PA)

PACS \#1: 43.25.Dc Nonlinear acoustics of solids

PACS \#2: 43.25.Rq Solitons, chaos

PACS \#3: 43.25.Vt Intense sound sources 